\title{
Do tyrosine kinase inhibitors lose favor in treatment of first-line metastatic renal cell carcinoma?
}

\author{
Samet Yaman1, Burak Bilgin², Mehmet AN Şendur*,2, Mutlu Hızal², Muhammed Bülent \\ Akıncıㄹ \& Bulent Yalçın² \\ ${ }^{1}$ Department of Internal Medicine, Ankara Yıldırım Beyazıt University, Ankara, Turkey \\ ${ }^{2}$ Department of Medical Oncology, Ankara Yıldırım Beyazıt University, Ankara, Turkey \\ *Author for correspondence: Tel.: +90 312 2912525; Fax: +90 312 2912726; masendur@yahoo.com.tr
}

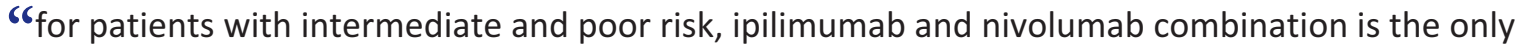
therapy with significant OS benefit compared with sunitinib and can be accepted as a new standard therapy, however cabozantinib may be a suitable option for these patients."

First draft submitted: 6 July 2018; Accepted for publication: 7 January 2019; Published online: 11 March 2019

Keywords: immunotherapy • renal cell carcinoma • tyrosine kinase inhibitors

Renal cell carcinoma (RCC) is the most common cancer of the kidneys. In USA, an estimated 40,080 and 23,380 new cases occurred in males and females in 2017, respectively. In addition, an estimated 14,400 patients died from RCC in 2017 [1]. The most common subtype of RCC is clear cell variant and also several distinct subtypes of RCCs have been identified, including papillary (chromophilic), chromophobe, oncocytic and collecting duct (Bellini's duct). The most common genetic alteration of clear cell variant RCC is the deletion of chromosome $3 \mathrm{p}$ which contains several genes associated with RCC, including the Von Hippel-Lindau (VHL). Results of VHL gene inactivation, HIF1A is increased and consequently, VEGF and PDGF- $\beta$ are overexpressed [2]. In addition to VEGF, AKT and mTOR signal pathways that stimulated to angiogenesis are also activated as a result of increased HIF1A level [3]. Antiangiogenic agents including tyrosine kinase inhibitors (TKIs) which targeted to VEGFR in RCC therapy are used in first and subsequent lines. It is also known that RCC is the one of the malignancy that had high mutational load and increased neoantigens expression. Therefore, clinical trials that investigated to efficacy of the checkpoint inhibitors in metastatic RCC were initiated and results of combination of the checkpoint inhibitors were published recently.

Sunitinib malate is an orally administered inhibitor of tyrosine kinases, including VEGFR and PDGF- $\beta$. In 2007, an international, multicenter and randomized Phase III trial that investigated sunitinib as a first-line treatment compared with IFN- $\alpha$ was published [4]. In this trial, sunitinib was administered orally at a dose of $50 \mathrm{mg}$ once daily, in 6-week cycles consisting of 4 weeks of treatment followed by 2 weeks without treatment. A total of 750 patients were enrolled and randomized to sunitinib or IFN- $\alpha$ arms. The primary end point of the study was progression-free survival (PFS) and secondary end points were also objective response rate (ORR) and overall survival (OS). The ORR was found higher in sunitinib arms than IFN- $\alpha$ (31 vs 6\%; $\mathrm{p}<0.001$ ). Median PFS was also longer in sunitinib arm compared with IFN- $\alpha$ (11 vs 5 months, hazard ratio [HR]: 0.42; 95\% CI: 0.32-0.54; p < 0.001). In subgroups analyzed, sunitinib is also more effective than IFN- $\alpha$ in good-, intermediate- and poor-risk patient groups according to Memorial Sloan Kettering Cancer Center risk classification. Updated results of this trial were published in 2009 [5] and median OS (mOS) was found to be higher in the sunitinib group than in IFN group (26.4 vs 21.8 months; HR: $0.821 ; \mathrm{p}=0.051$ ). Sunitinib is generally tolerated, and grade 3 and 4 adverse events were relatively low in both groups. The most common grade 3-4 toxicity of sunitinib were hypertension (8\%), fatigue $(7 \%)$, diarrhea (5\%), neutropenia (11\%), thrombocytopenia (8\%), lymphopenia (12\%) and increased lipase $(13 \%)$. 
Pazopanib is an oral angiogenesis inhibitor targeting VEGFR, PDGFR and c-Kit. The first randomized, doubleblind, placebo-controlled Phase III study of pazopanib monotherapy in treatment-naive and cytokine-pretreated patients with advanced and/or metastatic RCC was published in 2010 [6]. A total of 435 patients enrolled and were randomly assigned in a 2:1 ratio to receive either $800 \mathrm{mg}$ pazopanib or placebo. The PFS was significantly longer in pazopanib compared with placebo in the overall study population (median PFS: 9.2 vs 4.2 months; HR: 0.46; 95\% CI: 0.34-0.62; $\mathrm{p}=0.0001$ ). In subgroup analyses, PFS was also longer in treatment-naive population with 11.1 months for pazopanib and 2.8 months for placebo (HR: 0.40; 95\% CI: $0.27-0.60 ; p=0.0001$ ). The ORR was found as $30 \%$ in pazopanib group. A randomized, open-label, Phase III noninferiority COMPARZ trial that compared pazopanib with sunitinib in first-line metastatic RCC was published in 2013 [7]. Median PFS and OS were similar and found to be noninferior for pazopanib compared with sunitinib (median PFS: 8.4 months pazopanib group and 9.5 months sunitinib group; HR: 1.05). The grade 3 and 4 adverse events occurred more frequently in sunitinib than pazopanib groups. The most common grade 3 and 4 adverse event fatigue, hand-foot syndrome, lymphocytopenia, hypophosphatemia, increased aspartate aminotransferase (AST) and alanine aminotransferase (ALT).

Cabozantinib is another oral inhibitor of VEGFR 2, MET and AXL. In Phase II CABOSUN trial that investigated efficacy and safety of cabozantinib in first-line metastatic RCC was published in 2017 [8]. In this trial, patients classified as intermediate and poor risk categories according to The International Metastatic Renal Cell Carcinoma Database Consortium were enrolled and randomized to cabozantinib or sunitinib arms. Median PFS was significantly higher in cabozantinib group compared with sunitinib, (8.2 vs 5.6 months) and cabozantinib was associated with $34 \%$ risk reduction of progression or death (HR: $0.66 ; 95 \%$ CI: $0.46-0.95 ; \mathrm{p}=0.012$ ). The ORR was also higher in cabozantinib than sunitinib (33 vs 12\%, respectively). The grade 3 or 4 adverse events were similar between two groups (67\% for cabozantinib and $68 \%$ for sunitinib groups). An updated result of this trial was also published recently [9]. According to updated results, mOS tend to be better in cabozantinib arm (26.6 months for cabozantinib and 21.2 months for sunitinib) but this OS difference was not statistically significant (HR: 0.80; 95\% CI: 0.53-1.21).

Axitinib is a potent and selective inhibitor of VEGFR. The randomized Phase III trial that investigated to axitinib, a potent and selective inhibitor of VEGFR, in treatment-naive patients with metastatic RCC was published in 2013 [10]. Median PFS was numerically longer in axitinib arms but this difference was not statistically significant (10.1 vs 6.5 months; HR: 0.77; $\mathrm{p}=0.038$ ). In updated results, $\mathrm{mOS}$ was found similar between two groups with 21.7 months for axitinib and 23.3 months for sorafenib (HR: 0.99; $p=0.48$ ).

Nivolumab is an immune checkpoint inhibitor that blocks the interaction between PD-1 and PD-L1 and PD-L2, and previously trials showed that nivolumab had superior outcome in patients with progressed after prior antiangiogenic treatment. Ipilimumab is another immune checkpoint inhibitor that inhibits interaction between CTLA- 4 and B7. The CheckMate 214 trial that investigated efficacy and safety of nivolumab plus ipilimumab compared with sunitinib in advanced RCC was published in April 2018 [11]. This was a randomized, open-label Phase III trial of nivolumab plus ipilimumab followed by nivolumab monotherapy versus sunitinib monotherapy. Primary end points of this trial were PFS, ORR and OS. In this trial, patients who were classified as intermediate and poor risk categories according to The International Metastatic Renal Cell Carcinoma Database Consortium, were enrolled and randomized to nivolumab plus ipilimumab followed by nivolumab monotherapy or sunitinib arms. The OS was significantly higher in nivolumab plus ipilimumab group compared with sunitinib group in patients with intermediate- and poor-risk categories (12-month OS rate was 80 vs $72 \%$ and 18 -month OS was 75 vs $60 \%$; HR for death: 0.63 ; $p<0.001$ ). Nivolumab plus ipilimumab had higher ORR with $42 \%$ (complete response rate: $9 \%$, partial response rate: $32 \%)$ than sunitinib group with $27 \%(\mathrm{p}<0.001)$. The PFS was also superior in combination arm with 11.6 months for nivolumab plus ipilimumab and 8.4 months for sunitinib arms, but was not statistically significant ( $\mathrm{p}=0.03$, prespecified threshold was determined as 0.009$)$. In subgroup analyses according to PD-L1 expression level, patients with PD-L1 expression $\geq 1$ had better outcomes than PD-L1 expression $<1$. The 12-month OS rate was 86 and $80 \%$ and ORR was 58 and 37\% in PD-L1 expression level $\geq 1$ and $<1$ subgroups, respectively. Grade 3 and 4 adverse events in nivolumab plus ipilimumab and sunitinib occurred in 46 and 63\% of patients, respectively. Treatment-related adverse events leading to discontinuation occurred in $22 \%$ of patients in the nivolumab plus ipilimumab arm.

Preliminary result of the IMmotion 151 trial which investigated efficacy of atezolizumab and bevacizumab in treatment-naive RCC was also published in ASCO-GU 2018 [12]. In this trial, patients were randomized to atezolizumab plus bevacizumab (arm A) and sunitinib (arm B) and stratified according to PD-L1 status. Median 
PFS was 11.2 months for arm A and 7.7 months for arm B in PD-L1-positive patients (HR: 0.74; p = 0.02). Median PFS was also found better in ITT population (11.2 vs 8.2 months; HR: 0.83). The PFS was also better in all Memorial Sloan Kettering Cancer Center risk groups. The OS data of this trial are not mature yet and are still pending.

The VEGF has an important role in pathogenesis of RCC and TKI which targeted to VEGF is a principle treatment approach in first-line metastatic RCC until today. In addition, recently published Checkmate 214 and IMmotion 151 trials showed that the combination of nivolumab and ipilimumab and also the combination of atezolizumab and bevacizumab are superior to sunitinib in first-line metastatic RCC. According to subgroup analyses of Checkmate 214 trial, despite nivolumab plus ipilimumab having higher OS and ORR in intermediateand poor-risk categories, sunitinib had numerically superior OS, PFS and ORR than combination arm in the good-risk category. In addition, according to preliminary results of the IMmotion 151 trial, atezolizumab plus bevacizumab are superior in all MKSCC risk groups within the PD-L1-positive subgroup. Nowadays, sunitinib or pazopanib are still the optimal first-line treatment approach in patients with good risk. However, atezolizumab plus bevacizumab and nivolumab plus ipilimumab are superior to sunitinib intermediate- and poor-risk with acceptable adverse events profile. In addition, cabozantinib which targeted VEGFR, AXL and MET also has a significantly superior outcome compared with sunitinib in patients with intermediate and poor risk, in Phase II CABOSUN trial. According to results of these trials, for patients with intermediate and poor risk, ipilimumab and nivolumab combination is the only therapy with significant OS benefit compared with sunitinib and can be accepted as a new standard therapy, however cabozantinib may be a suitable option for these patients. Nowadays, there are many ongoing trials investigating the combination of checkpoint inhibitors and TKI which targeted VEGFR. The optimal first-line treatment of RCC may change after the completion of these ongoing trials.

\section{Financial \& competing interests disclosure}

The authors have no relevant affiliations or financial involvement with any organization or entity with a financial interest in or financial conflict with the subject matter or materials discussed in the manuscript. This includes employment, consultancies, honoraria, stock ownership or options, expert testimony, grants or patents received or pending, or royalties.

No writing assistance was utilized in the production of this manuscript.

\section{References}

1 Siegel RL, Miller KD, Jemal A. Cancer statistics, 2017. CA Cancer J. Clin. 67, 7-30 (2017).

2 Kim WY, Kaelin WG. Role of VHL gene mutation in human cancer. J. Clin. Oncol. 22, 4991-5004 (2004).

3 Hudson CC, Liu M, Chiang GG et al. Regulation of hypoxia-inducible factor 1 alpha expression and function by the mammalian target of rapamycin. Mol. Cell. Biol. 22, 7004-7014 (2002).

4 Motzer RJ, Hutson TE, Tomczak P et al. Sunitinib versus interferon alfa in metastatic renal-cell carcinoma. N. Engl. J. Med. 356, 115-124 (2007).

5 Motzer RJ, Hutson TE, Tomczak P et al. Overall survival and updated results for sunitinib compared with interferon alfa in patients with metastatic renal cell carcinoma. J. Clin. Oncol. 27, 3584-3590 (2009).

6 Sternberg CN, Davis ID, Mardiak J et al. Pazopanib in locally advanced or metastatic renal cell carcinoma: results of a randomized Phase III trial. J. Clin. Oncol. 28, 1061-1068 (2010).

7 Motzer RJ, Hutson TE, Cella D et al. Pazopanib versus sunitinib in metastatic renal-cell carcinoma. N. Engl. J. Med. 369, 722-731 (2013).

8 Choueiri TK, Halabi S, Sanford BL et al. Cabozantinib versus sunitinib as initial targeted therapy for patients with metastatic renal cell carcinoma of poor or intermediate risk: the Alliance A031203 CABOSUN trial. J. Clin. Oncol. 35, 591-597 (2017).

9 Choueiri TK, Hessel C, Halabi S et al. Cabozantinib versus sunitinib as initial therapy for metastatic renal cell carcinoma of intermediate or poor risk (Alliance A031203 CABOSUN randomised trial): progression-free survival by independent review and overall survival update. Eur. J. Cancer 94, 115-125 (2018).

10 Hutson TE, Al-Shukri S, Stus VP et al. Axitinib versus sorafenib in first-line metastatic renal cell carcinoma: overall survival from a randomized Phase III trial. Clin. Genitourin. Cancer 15, 72-76 (2017).

11 Motzer RJ, Tannir NM, McDermott DF et al. Nivolumab plus ipilimumab versus sunitinib in advanced renal-cell carcinoma. N. Engl. J. Med. 378, 1277-1290 (2018).

12 Motzer RJ, Powles T, Atkins MB et al. IMmotion 51: a randomized Phase III study of atezolizumab plus bevacizumab vs sunitinib in untreated metastatic renal cell carcinoma (mRCC). J. Clin. Oncol. 36 (6 Suppl.), 578 (2018). 